\title{
MicroRNAs in autoimmune disease
}

\author{
Vivek K. Rauniyar $\cdot$ Wei Wu Huan Yang
}

Received: 21 March 2011/Accepted: 21 June 2011/Published online: 12 July 2011

(c) Springer-Verlag 2011

\begin{abstract}
MicroRNAs (miRNAs) are non-coding, singlestranded small RNAs, usually 18-25 nucleotides long, have ability to regulate gene expression post-transcriptionally. miRNAs are highly homologous, conserved and are found in various living organisms including plants and animals. Present studies show that these small RNAs anticipate and are directly involved in many important physiological and pathological processes including growth, proliferation, maturation, metabolism, and inflammation among others. Evidences are accumulating that miRNAs play active role in directing immune responses and, therefore, might be involved in pathogenesis of autoimmune diseases. Recent studies have found that miRNAs are critical in proliferation, maturation and differentiation of T cells, B cells and, therefore, may affect the outcome of an immune response. In light of such understanding, this review briefly introduces miRNAs and discusses its role in the pathogenesis of various autoimmune diseases, as well as its potential as a biomarker and therapeutic target in the management of autoimmune diseases.
\end{abstract}

Keywords MicroRNA - Immune system - T cells · B cells $\cdot$ Autoimmune diseases

\section{Introduction}

MicroRNAs (miRNAs), a class of highly conserved, small, and non-coding RNA molecules of usually 18-25

V. K. Rauniyar · W. Wu · H. Yang $(\bowtie)$ Department of Neurology, Xiangya Hospital, Xiangya School of Medicine, Central South University, 87 th Xiangya road, Changsha 410008, Hunan, China e-mail: huany@ymail.com nucleotides (nt) in length [1], have attracted attention of the scientific community worldwide in recent years. These minute RNAs, as the name implies, are thought to play critical roles in a variety of physiological and pathological processes in the plants and animals. miRNAs act on the protein synthesis machinery of a cell through negative post-transcriptional modification/regulation of messenger RNAs (mRNAs) by either blocking its translation and/or affecting its stability $[1,2]$. Their first discovery was made in Caenorhabditis elegans [3]; since then numerous miRNAs have been identified. Mirbase, which index the latest and comprehensive data on miRNAs, shows that 1,788 miRNAs have been discovered in human beings alone so far [4]. Researchers previously estimated that the human genome might encode more than 1,000 miRNAs and it has turned out to be true as the discovery of the new ones are still being made. In this review, we first introduce the miRNAs and later discuss on their possible roles in pathogenesis of some common autoimmune diseases.

\section{Biogenesis and function overview}

MicroRNAs participate and regulate some of the most basic cellular physiological processes including proliferation, differentiation, maturation, apoptosis, metabolism and also modulate immune responses and inflammation required for normal functioning of the cells and organism [1, 5-7].

In nucleus, primary microRNAs (Pri-miRNAs) transcripts are transcribed by RNA polymerase II [8] and are cleaved into 70-100 nt long hairpin loop structure known as pre-microRNAs by DROSHA/DGCR8, a RNA polymerase III enzyme complex. Pre-miRNAs are actively transported to the cytoplasm by exportin [9], a nuclear membrane transporter, and are again cleaved to $18-25 \mathrm{nt}$ 


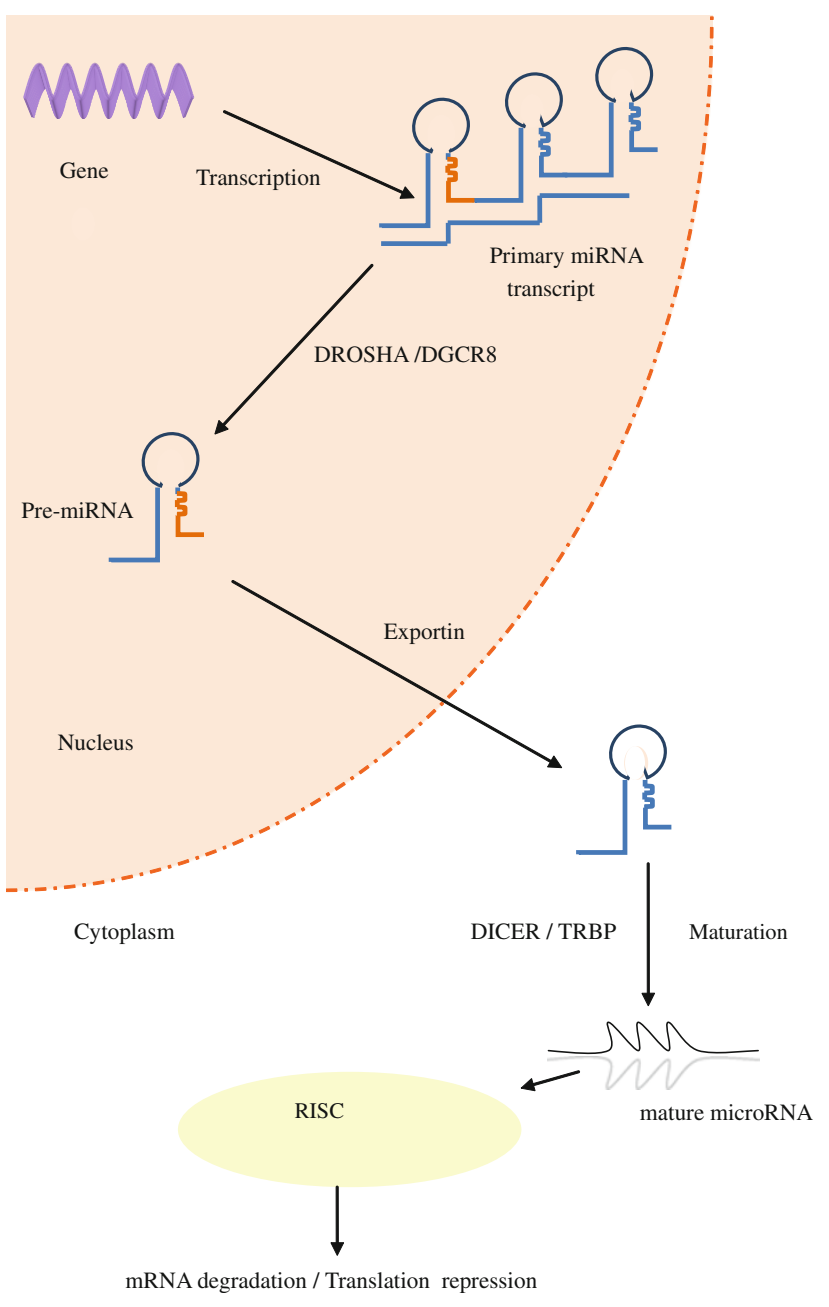

Fig. 1 Biogenesis and function of microRNA

double stranded microRNAs by a RNA polymerase III, DICER/TRBP enzyme complex, present in the cytoplasm. One of the strands of these double stranded RNA forms a mature microRNA (MiRNA), which is loaded on RNAinduced silencing complex (RISC) also containing argonaute proteins, and target messenger RNAs (mRNAs) $3^{\prime}$ untranslated region ( $3^{\prime}$ UTR) eventually resulting in mRNAs degradation and/or translation repression [10] (Fig. 1). This targeting of mRNAs brings about modulation and 'fine-tuning' of the protein synthesis and its effects.

\section{MicroRNA detection}

MicroRNAs, being very small RNAs, are difficult to detect and isolate from biological tissues. But techniques for detection and quantification have steadily improved in the last few years. Among them northern blotting, microarrays and polymerase chain reaction (PCR) have enjoyed widespread use [11]. These methods are applicable to almost all types of tissues, body fluids for the detection of microRNAs. Northern blotting, in particular, is referred as 'gold standard' method for isolation and quantification [11]. But, given its difficulties and complexities, microarrays and real-time quantitative PCR have been commonly used in the laboratories for profiling of microRNAs and have been more popular [11]. All three methods have their own pros and cons. Recently, several other methods including, in particular, locked nucleic acid modified probe fluorescence in situ hybridization (LNA-FISH) has been used with good results as a newer technique in microRNA detection [12]. Overall, newer methods and techniques have been continuously developed and modified, which have helped in better detection and quantification of microRNAs and this will undoubtedly further enrich our understanding of microRNAs.

\section{MicroRNAs and immune-regulation}

Immune regulation is a complex but finely regulated process. Maintenance of the tolerance and elimination of the foreign microbes are major facets of this big picture, which effectively maintain the immune homeostasis. Analysis of miRNAs expression in the murine hematopoietic system has showed important general effects on the mechanism of cell differentiation and maintenance of cell identity. miRNAs have been found to play essential roles in the differentiation of immune cells including $\mathrm{T}$ cells, B cells, and antigen-presenting cells among others [7]. miR-181a, miR-150, and miR-17-92 cluster regulate the differentiation and development of the early phase of both B and T cells from haematopoietic stem cells [13, 14]. Similarly, miR-223 is required for differentiation of various myeloid cells including neutrophils, basophils, and mast cells from common myeloid precursor cells [15]. miRNAs also regulate various steps in innate and acquired immune responses. Several microRNAs including miR-155, miR-146, miR-132, and miR-223 regulate the acute inflammatory response [16-19]. Our understanding of the roles of miRNAs in immune response primarily come from transgenic animal models especially deletion of dicer in various immune cells including $\mathrm{T}$ and $\mathrm{B}$ cells $[20,21]$ and complemented with observations in human subjects.

miR-181a was one of the first miRNAs discovered to have a role in immune cell development. miR-181a regulates p27 mRNA translation during myeloid cell differentiation [22]. Likewise, miR-181a was found to be highly expressed in the thymus, and was detectable in the spleen and bone marrow progenitors, and in lower level in $\mathrm{B} 220^{+}$ B cells [23]. Moreover, ectopic expression of miR-181a in hematopoietic stem/progenitor cells lead to an increase in $\mathrm{B}$ cells and decrease in $\mathrm{T}$ cells, especially $\mathrm{CD}^{+} \mathrm{T}$ cells. 
However, it has also been noted that miR-181a may promote $\mathrm{CD}^{+}{ }^{+} \mathrm{CD}^{+} \mathrm{T}$ cells development when ectopically expressed in thymic progenitor cells [24]. Studies have revealed that miR-181a may regulate $\mathrm{T}$ cells through targeting Bcl-2, CD69 and TCR [22-24] or modulating the sensitivity of $\mathrm{T}$ cells to antigen. Overall, miR-181a regulates thymocytes development, modulates TCR signaling and regulates $B$ cell differentiation [6, 23-25].

Similarly, miR-155 has received a lot of attention in the recent studies. It is a process from an exon of the noncoding RNA, bic [26]. Activated B cells, T cells, antigen presenting cells including macrophages and dendritic cells express miR-155 in increased levels [16]. It has also been found to be over expressed in B cell lymphomas, Hodgkin's disease [27, 28] and some solid tumors [26]. This fact is also corroborated by the other studies which show that over expression of miR-155 leads to B cell malignancies in transgenic murine models [29]. These results are highly suggestive of miR-155 involvement in crucial steps of B cell differentiation and proliferation. Further consolidating the findings, studies on miR-155 knockout mice demonstrated that miR-155 plays an essential role in the regulation of both $\mathrm{T}$ and $\mathrm{B}$ cells during the acquired immune response. This was evidenced by the observations that the mice showed inability to mount an immune response to pathogens following appropriate immunization [30] despite being healthy otherwise. Moreover, in the acute immune response phase, $\mathrm{T}$ cells from miR-155 knockout mice exhibited attenuated IL- 2 and IFN- $\gamma$ release in response to antigenic stimulation [31]. In addition, $\mathrm{CD} 4^{+} \mathrm{T}$ cells from these mice were intrinsically biased towards $\mathrm{T}_{\mathrm{H}} 2$ differentiation, with concomitant production of $\mathrm{T}_{\mathrm{H}} 2$ cytokines $[31,32]$. Similarly, other studies also demonstrated that miR-155 may have a potential role in regulating the development and function of regulatory $\mathrm{T}$ cells (Tregs) [33]. Interestingly, it was found that although miR-155 knockout mice had normal circulating B cell counts, they were found to have reduced numbers of germinal centre B cells, thus explaining the immunodeficiency. Taken together, miR-155 seems to be very essential for B and $\mathrm{T}$ cells functions both for normal development and mounting immune response. The magnitude of the effects on $\mathrm{T}$ and $\mathrm{B}$ cells needs to be thoroughly studied in detail in future studies so as to elucidate and establish the exact role and place of miR-155 in the immune system.

Similarly, miR-146 has been found to be induced in response to exposure of human monocytic THP-1 cell lines and dendritic cells to bacterial lipo-polysaccharides and IL-1, TNF-alpha [16]. Interleukin-1 receptor-asscoiated kinase 1 (IRAK1) and TNF receptor-associated factor 6 (TRAF6) have been discovered as the targets of miR-146 and are downregulated with co-expression of miR-146. As it is known that TRAF6 is crucial in nuclear factor $\mathrm{kB}$
(NF-kB) activation, it seems that miR-146 is important negative regulator of NF-kB mediated inflammatory pathway. Interestingly it has also been found to be expressed in high amounts in regulatory $\mathrm{T}$ cells and $\mathrm{T}_{\mathrm{H}} 1$ cells. More studies would be required to unravel its role in detail in the immune response.

Likewise, miR17-92 cluster has been found to be required for transition of $B$ cell from pro- to pre-B cells [34]. Moreover, it is required for T cell transition from pro-T cell to pre-T cell. Pro-apoptotic pathways involving Bim and PTEN (phosphatase and tensin homolog) are found to be the targets of the miR-17-92, where it suppress expression of Bim protein especially in pro-B cells and antagonize apoptosis [34].

Recently, it has been discovered that miR-182 is essential for the activation and development of $\mathrm{T}$ helper cells. miR-182 is induced by IL-2 and inactivates the transcription factor Foxo1 which is required for the clonal expansion and further development of $\mathrm{T}$ helper cells in IL-2 dependent pathway [35].

\section{MicroRNAs and autoimmune diseases}

\section{MicroRNAs in SLE and ITP}

Systemic lupus erythematosus (SLE) is a systemic inflammatory autoimmune disease characterized by autoantibodies against various self-antigens and damage to major organ systems. The exact cause of SLE remains unexplained though both genetic predispositions and environmental influences [36] have been thought to play roles in the pathogenesis of the disease. Many studies on the potential relationship between miRNAs and SLE have not been conducted so far. A study conducted by Dai et al. [37] examined and compared 35 miRNAs expression in PBMCs among SLE patients, idiopathic thrombocytopenic purpura (ITP) patients and healthy controls. It was revealed that 28 miRNAs were significant in their expression levels of which 7 miRNAs were down-regulated and 9 miRNAs were up-regulated in SLE group. Moreover, it was observed that some miRNAs were directly related with disease activity. In addition, a negative correlation was discovered between SLE disease activity index (SLEDAI) and eight miRNAs. This might imply direct involvement of miRNAs in the pathogenesis of SLE and has further fuelled research on the topic. Moreover, the study provided evidence of involvement of the miRNAs in the progress, recrudescence and organ injury of SLE.

Idiopathic thrombocytopenic purpura (ITP) is an autoimmune disorder characterized by a low platelet count and mucocutaneous bleeding [38, 39] and occurs in all ages, in acute and chronic forms. In the above mentioned study, 19 
related miRNAs were noted, of which 14 miRNAs were down-regulated and 5 miRNAs were up-regulated. Interestingly, 13 miRNAs were found to have the same expression patterns both in SLE and ITP. Though it might be premature to conclude, but these results demonstrated that these miRNAs might be associated with autoimmune process in general. Further detailed studies are needed to clarify above results. The second group demonstrated three miRNAs, all of which up-regulated or down-regulated in SLE with no significant changes observed in ITP. These three miRNAs yet might be a part of or engaged in systemic inflammatory response. The third group which comprised of six miRNAs was noted to be down-regulated in ITP only. These miRNAs might be correlated with the organ specific destruction of thrombocytes. Evidently, the study has established the presence of miRNAs in ITP but further studies would clear their roles in the disease.

Again another interesting study was led by Dai et al. [40] on miRNAs and lupus nephritis (LN). miRNAs of renal biopsied, LN patients and healthy controls were isolated and analysed. The study identified 66 miRNAs in renal samples, of which 30 miRNAs were down-regulated and 36 miRNAs were up-regulated in LN biopsies.

A comprehensive study conducted by Tang et al. [41] revealed that under expression of miR-146a might contribute to over activation of the type I interferon (IFN) pathway in lupus patients. Analysis of the results of the study unveiled that there were seven miRNAs down-regulated evidently in SLE patients when compared with controls. Among these miRNAs, miR-146a had already been reported to negatively regulate the innate immune response [42-44]. To verify this fact, miR-146a expression was examined in a larger group of SLE patients and controls which yielded significantly lower expression of miR-146a in lupus patients when compared with normal controls. Moreover, miR-146a expression levels also correlated negatively with SLE disease activity, SLEDAI scores, and renal SLEDAI scores. Further study demonstrated that miR-146a appeared to regulate the immune activation by targeting multiple key signaling proteins in the type I IFN pathway, thus inhibiting the exaggerated immune response. When miR-146a was introduced in PBMCs of the SLE patients, the coordinate activation of the type I IFN pathway was notably reduced, as revealed by down-regulation of several IFN-inducible genes. These results suggested that the manipulation of miR-146a levels could potentially provide a therapeutic benefit to lupus patients.

Taken together, studies effectively demonstrated that miRNAs might play important role in the development of SLE, both during immune regulation and organ injury processes. The findings also suggest that miRNAs could be a potential biomarker of the disease and might serve as potential therapeutic targets for the treatment as well.
Further detailed studies are required to confirm and clarify the implications of the above observations.

\section{MicroRNAs in rheumatoid arthritis}

Rheumatoid arthritis (RA) is a systemic autoimmune disorder characterized by chronic inflammation of synovial tissue that results in irreversible joint damage [45]. Inflammatory cytokines, including tumor necrosis factor (TNF)- $\alpha$ and IL-1 $\beta$ have been indentified to play crucial roles in the pathogenesis of the disease, and inhibition of these cytokines could ameliorate the disease at least in subset of patients $[46,47]$. Recent studies have profiled the expression pattern of miRNAs in RA patients. miR-155, and miR-146a expressions have been found to be upregulated in RA synovial fibroblasts [48]. Moreover, miR-155 expression was higher in RA synovial fluid monocytes as compared to RA peripheral blood mononuclear cells (PBMCs). This might be suggestive of miR-155 role in modulating downstream tissue damage. Similarly, PBMCs obtained from RA patients exhibited increased expression of miR-146a, miR-155, miR-132 and miR-16 [49], whereas no significant difference was noted for let-7a expression. Furthermore, high expression levels of miR-14a, and miR-16 correlated with disease activity, and low expression levels correlated with inactive disease. These data provide preliminary evidence of the role of micro-RNAs in RA and suggest that miR-146a and miR-16 expression levels may be a used as marker of RA disease activity.

Likewise, miR-124a level was found significantly decreased in RA synoviocytes when compared with OA (osteoarthritis) synoviocytes [50]. Transfection of precursor miR-124a into RA synoviocytes significantly suppressed their proliferation and arrested the cell cycle at the $\mathrm{G}_{1}$ phase. miRanda 3.0 was used to search for putative miR-124a targets. Analysis of the results and other experiments suggested cycling-dependent kinase-2 (CDK-2) and monocyte chemoattractant protein 1 (MCP-1) mRNA as the targets of miR-124a. And it was noted that over expression of miR-124a substantially suppress the expression of CDK-2 and MCP-1.

In another interesting study [51], miR-15a was found significantly down-regulated in murine model of induced arthritis. In addition, the Bcl-2 protein, which had been demonstrated as the direct target of miR-15a, was also confirmed to be up-regulated in arthritic mice. To verify and observe the effects of miR-15a, the mice in the experimental group were injected intra-articularly with double-stranded miR-15a-atelocollagen complex and the control group with siRNA-atelocollagen complex. After confirming that the double-straded miR-15a had been taken up by the cells in the synovium, the researchers evaluated the immunohistochemistry of caspase 3 in order to examine 
cell apoptosis. The expression of caspase 3 was found to be increased in the synovium of mice in the experimental group, while the expression of caspase 3 could not be observed in the control group. The study successfully induced cell apoptosis by the intraarticular injection double-straded miR-15a. This study opens the door for the possibility of a new treatment strategy in RA.

Another study identified that mature miR-146a and primary miR-146a/b were highly expressed in RA synovial tissue along with TNF- $\alpha$ [52]. Cells positive for miR-146a in synovial tissue were primarily $\mathrm{CD}^{+} 8^{+}$macrophages, but also included several $\mathrm{CD}^{+} \mathrm{T}$ cell subsets and $\mathrm{CD}^{+} \mathrm{a}^{+} \mathrm{B}$ cells. Expression of miR-146a was remarkably up-regulated in RA synovial tissue after stimulation with TNF- $\alpha$ and IL- $1 \beta$. The results of the study might be suggestive of miR-146a function in the termination of inflammation triggered by $\mathrm{TNF}-\alpha$ and IL- $1 \beta$.

\section{MicroRNAs in multiple sclerosis}

Multiple sclerosis (MS) is a demyelinating disease of the central nervous system. miRNAs might play role in the development and pathogenesis of MS. hsa-miR-96, might be characteristic of the remitting phase of MS [53]. A gene ontology analysis with the target genes of miR-96 demonstrated that miR-96 is involved in the interleukin signaling pathways and other pathways. These pathways are concerned with activation and regulation of T-cells [54, 55]. Similarly, study suggested that hsa-miR-18b and hsa-miR599 might be involved in the relapsing phase of MS.

Recently, Keller et al. [56] identified 165 significantly dysregulated miRNAs in relapsing-remitting MS (RRMS) patients when compared with healthy controls. Among these, 74 miRNAs were up-regulated and 91 were downregulated. Significantly dysregulated miRNAs were hsamiR-145, hsa-miR-186, hsa-miR-664, hsa-miR-20b, hsa-miR-442a, hsa-miR-142-3p, hsa-miR-584, hsa-miR-223, hsa-miR-1275 and hsa-miR-491-5p, altogether ten of them. Interestingly, nine of them were significantly up-regulated, while only hsa-miR-20b was down-regulated. Comparing the results of the studies, seven miRNAs that had high information content were identified and among these four miRNAs, hsa-miR-509-3-5p, hsa-miR-34c-3p, has-miR214, and hsa-miR-509-5p were highly expressed in healthy individuals when compared with MS patients whereas miRNAs, hsa-miR-328, hsa-miR-30a and hsa-miR-30e were significantly expressed in PBMCs of MS patients.

Yet in another interesting study, Du et al. [57] tried to explore the relationship between miR-326 and $\mathrm{T}_{\mathrm{H}^{-}} 17$ in MS. Interleukin 17(IL-17)-producing $\mathrm{T}$ helper cells, $\mathrm{T}_{\mathrm{H}^{-1}} 17$ cells, have been identified as distinct subset of effector helper $\mathrm{T}$ cells essential in clearing extracellular pathogens and responsible for inducing tissue inflammation in various autoimmune diseases including MS [58]. Study investigated miR-326 as a $\mathrm{T}_{\mathrm{H}^{-}} 17$ cell-associated miRNA which revealed that the expression of miR-326 in $\mathrm{CD}^{+}{ }^{+} \mathrm{T}$ cells highly correlated with disease severity in MS patients and EAE mice, the animal model of $\mathrm{M}$. The study was first to suggest that miR-326 might be critically involved in the pathogenesis of $\mathrm{MS}$ by regulating $\mathrm{T}_{\mathrm{H}^{-}} 17$ differentiation. Later on, it was also demonstrated that by targeting Est-1, a known negative regulator of $\mathrm{T}_{\mathrm{H}^{-}}-17$ cells, miR-326 could promote the generation of $\mathrm{T}_{\mathrm{H}^{-}} 17$ cells both in vivo and in vitro [57].

As evidenced by the study, miR-326 might play an important role in $\mathrm{T}_{\mathrm{H}} 17$ differentiation and thus the pathogenesis of MS. Besides these, very few studies have investigated miRNAs expression in MS patients in such detail and their roles in the development and pathogenesis of the disease remains obscure. Further studies are needed in order to ascertain the identification of the dysregulated miRNAs in MS.

\section{Conclusion and perspective}

MicroRNAs seem to play crucial roles in the normal functioning of the immune system and the pathogenesis of autoimmune diseases. Certain miRNAs are closely related with the development and severity of autoimmune diseases while some of them are involved in the activation, regulation of immunocytes, immune responses and still others anticipate the organ injury process. But the precise mechanism by which miRNAs affect autoimmune diseases still remains elusive for now, and further elaborative studies using transgenic animal models along with observations in human subjects would be needed to appreciate the whole picture. In the light of current understanding, we emphasize that miRNAs have the potential to serve as biomarkers, as well as a new therapeutic target in the management of autoimmune diseases in the future.

Conflict of interest None.

\section{References}

1. He L, Hannon GJ (2004) MicroRNAs: small RNAs with a big role in gene regulation. Nat Rev Genet 5(7):522-531

2. Filipowicz W, Bhattacharyya SN, Sonenberg N (2008) Mechanisms of posttranscriptional regulation by microRNAs: are the answers in sight? Nat Rev Genet 9(2):102-114

3. Lee RC, Feinbaum RL, Ambros V (1993) The C. elegans heterochronic gene lin- 4 encodes small RNAs with antisense complementarity to lin-14. Cell 75:843-854

4. http://mirbase.org. Accessed March 2011

5. Bartel DP (2004) MicroRNAs: genomics, biogenesis, mechanism, and function. Cell 116(2):281-297 
6. Neilson JR, Zheng GX, Burge CB et al (2007) Dynamic regulation of miRNA expression in ordered stages of cellular development. Genes Dev 21:578-589

7. Monticelli S et al (2005) MicroRNA profiling of the murine hematopoietic system [J]. Genome Biol 6(8):R71

8. Lee $\mathrm{Y}$ et al (2004) MicroRNA genes are transcribed by RNA polymerase II. EMBO J 23:4051-4060

9. Yi R, Qin Y, Macara IG, Cullen BR (2003) Exportin-5 mediates the nuclear export of pre-microRNAs and short hairpin RNAs. Genes Dev 17:3011-3016

10. Chendrimada TP et al (2007) MicroRNA silencing through RISC recruitment of eIF6. Nature 447:823-828

11. Hunt EA, Goulding AM, Deo SK (2009) Direct detection and quantification of microRNAs. Anal Biochem 387(1):1-12

12. de Planell-Saguer M, Rodicio MC, Mourelatos Z (2010) Rapid in situ codetection of noncoding RNAs and proteins in cells and formalin-fixed paraffin-embedded tissue sections without protease treatment. Nat Protoc 5(6):1061-1073

13. Baltimore D, Boldin MP, O'Connell RM, Rao DS, Taganov KD (2008) MicroRNAs; new regulators of immune cell development and function. Nat Immunol 9(8):839-845

14. Zhou B, Wang S, Mayr C et al (2007) miR-150, a microRNA expressed in mature B and T cells, blocks early B cell development when expressed prematurely. Proc Natl Acad Sci USA 104:7080-7085

15. Fazi $\mathrm{F}$ et al (2005) A minicircuitry comprised of microRNA-223 and transcription factors NFI-A and $\mathrm{C} / \mathrm{EBP} \alpha$ regulates human granulopoiesis. Cell 123:819-831

16. Taganov KD, Boldin MP, Chang KJ, Baltimore D (2006) NFkappa B-dependent induction of microRNA miR-146, an inhibitor targeted to signaling proteins of innate immune responses. Proc Natl Acad Sci USA 103(33):12481-12486

17. O'Connell RM, Taganov KD, Boldin MP, Cheng G, Baltimore D (2007) MicroRNA-155 is induced during the macrophage inflammatory response. Proc Natl Acad Sci USA 104(5):16041609

18. Tili E et al (2007) Modulation of miR-155 and miR-125b levels following lipopolysaccharide/TNF-alpha stimulation and their possible roles in regulating the response to endotoxin shock. J Immunol 179(8):5082-5089

19. Johnnidis JB et al (2008) Regulation of progenitor cell proliferation and granulocyte function by microRNA-223. Nature 451: 1125-1129

20. Muljo SA et al (2005) Aberrant $T$ cell differentiation in the absence of Dicer. J Exp Med 202:261-269

21. Koralov SB et al (2008) Dicer ablation affects antibody diversity and cell survival in the B lymphocyte lineage. Cell 132:860-874

22. Cuesta R, Martínez-Sánchez A, Gebauer F (2009) miR-181a regulates cap-dependent translation of p27 (kip1) mRNA in myeloid cells. Mol Cell Biol 29(10):2841-2851

23. Chen CZ, Li L, Lodish HF et al (2004) MicroRNAs modulate hematopoietic lineage differentiation. Science 303:83-86

24. Liu G, Min H, Yue S et al (2008) Pre-miRNA loop nucleotides control the distinct activities of mir-181a-1 and mir-181c in early T cell development. PLOS ONE 3:e3592

25. Li Q et al (2007) miR-181a is an intrinsic modulator of $\mathrm{T}$ cell sensitivity and selection. Cell 129:147-161

26. Eis PS, Tam W, Sun L et al (2005) Accumulation of miR-155 and BIC RNA in human B cell lymphomas. Proc Natl Acad Sci USA 102:3627-3632

27. Calin GA, Croce CM (2006) MicroRNA signatures in human cancers. Nat Rev Cancer 6(11):857-866

28. Costinean $\mathrm{S}$ et al (2006) Pre-B cell proliferation and lymphoblastic leukemia/high-grade lymphoma in E(mu)-miR155 transgenic mice. Proc Natl Acad Sci USA 103(18):7024-7029
29. Fulci V, Chiaretti S, Goldoni M et al (2007) Quantitative technologies establish a novel microRNA profile of chronic lymphocytic leukemia. Blood 109:4944-4951

30. Rodriguez A, Vigorito E, Clare $S$ et al (2007) Requirement of bic/ microRNA-155 for normal immune function. Science 316: 608-611

31. Thai TH, Calado DP, Casola S et al (2007) Regulation of the germinal center response by microRNA-155. Science 316 : 604-608

32. Kohlhaas S, Garden OA, Scudamore C et al (2009) Cutting edge: the Foxp3 target miR-155 contributes to the development of regulatory $\mathrm{T}$ cells. J Immunol 182:2578-2582

33. Zheng Y, Josefowicz SZ, Kas A, Chu TT et al (2007) Genomewide analysis of Foxp3 target genes in developing and mature regulatory T cells. Nature 445:936-940

34. Ventura A et al (2008) Targeted deletion reveals essential and overlapping functions of the miR-17 through 92 family of miRNA clusters. Cell 132(5):875-886

35. Mashreghi MF et al (2010) The microRNA miR-182 is induced by IL- 2 and promotes clonal expansion of activated helper $\mathrm{T}$ lymphocytes. Nat Immunol 11(11):1057-1062

36. Yasutomo K, Horiuchi T, Kagami S et al (2001) Mutation of DNASE1 in people with systemic lupus erythematosus. Nat Genet 28:313-314

37. Dai Y, Huang Y-S, Tang M et al (2007) Microarray analysis of microRNA expression in peripheral blood cells of systemic lupus erythematosus patients. Lupus 16:939-946

38. McMillan R (1981) Chronic idiopathic thrombocytopenic purpura. N Engl J Med 304:1135-1147

39. George J, Harake M, Aster R (1995) Thrombocytopenia due to enhanced platelet destruction by immunologic mechanisms. In: Beutler E, Lichtman MA, Coller BS, Kipps TJ (eds) Williams hematology, 5th ed. edn. McGraw-Hill, New York, pp 1315-1355

40. Dai Y, Wang S, Lan H et al (2009) Comprehensive analysis of microRNA expression patterns in renal biopsies of lupus nephritis patients. Rheumatol Int 29:749-754

41. Tang Y, Luo X et al (2009) Micro-RNA-146a contributes to abnormal activation of the type $\mathrm{i}$ interferon pathway in human lupus by targeting the key signaling proteins. Arthr Rheum 60:1065-1075

42. Liew FY, Xu D, Brint EK, O'Neill LA (2005) Negative regulation of toll-like receptor-mediated immune responses. Nat Rev Immunol 5:446-458

43. Balaci L, Spada MC, Olla N et al (2007) IRAK-M is involved in the pathogenesis of early-onset persistent asthma. Am J Hum Genet 80:1103-1114

44. Fujimoto M, Tsutsui H, Xinshou O et al (2004) Inadequate induction of suppressor of cytokine signaling-1 causes systemic autoimmune diseases. Int Immunol 16:303-314

45. Smolen JS, Aletaha D, Koeller M et al (2007) New therapies for treatment of rheumatoid arthritis. Lancet 370:1861-1874

46. Bresnihan B, Varo-Gracia JM, Cobby M et al (1998) Treatment of rheumatoid arthritis with recombinant human interleukin-1 receptor antagonist. Arthr Rheum 41:2196-2204

47. Lipsky PE, Heijde DM, St Clair EW et al (2000) Infliximab and methotrexate in the treatment of rheumatoid arthritis. Anti-tumor necrosis factor trial in rheumatoid arthritis with concomitant therapy study group. N Engl J Med 343:1594-1602

48. Stanczyk J, Pedrioli DM, Brentano F, Sanchez-Pernaute O, Kolling C, Gay RE et al (2008) Altered expression of MicroRNA in synovial fibroblasts and synovial tissue in rheumatoid arthritis. Arthr Rheum 58:1001-1009

49. Kaleb MP et al (2008) Upregulated miR-146a expression in peripheral blood mononuclear cells from rheumatoid arthritis patients. Arthr Res Ther 10:R101 
50. Yuji N, Kawano S, Takenokuchi M et al (2009) MicroRNA-124a is a key regulator of proliferation and monocyte chemoattractant protein 1 secretion in fibroblast-like synoviocytes from patients with rheumatoid arthritis. Arthr Rheum 60:1294-1304

51. Yoshihiko N, Nakasa T, Mochizuki Y et al (2009) Induction of apoptosis in the synovium of mice with autoantibody-mediated arthritis by the intraarticular injection of double-stranded microrna-15a. Arthr Rheum 60:2677-2683

52. Nakasa T, Miyaki S, Okubo A et al (2008) Expression of MicroRNA-146 in rheumatoid arthritis synovial tissue. Arthr Rheum 58:1284-1292

53. Otargui D, Baranzini SE, Armananzas R et al (2009) Differential micro RNA expression in PBMC from multiple sclerosis patients. PLOS ONE 4(7):e6309

54. Vallejo-Illarramendi A, Domercq M, Perez-Cerda F, Matute C et al (2006) Increased expression and function of glutamate transporters in multiple sclerosis. Neurobiol Dis 21:154-164
55. Matute C (2007) Interaction between glutamate signalling and immune attack in damaging oligodendrocytes. Neuron Glia Biol 3:281-285

56. Keller A, Leidinger P, Lange J et al (2009) Multiple sclerosis: microRNA expression profiles accurately differentiate patients with relapsing-remitting disease from healthy controls. PLOS ONE 4(10): e7440

57. Du C, Liu C, Kang J et al (2009) MicroRNA miR-326 regulates $\mathrm{T}_{\mathrm{H}^{-}} 17$ differentiation and is associated with the pathogenesis of multiple sclerosis. Nat Immunol 10:1252-1259

58. Tesmer L, Lundy A, Sarkar SK, Fox DA (2008) Th17 cells in human disease. Immunol Rev 223:87-113 